\title{
Noncommutative M-branes from Covariant Open Supermembranes
}

\author{
Makoto Sakaguch* \\ Okayama Institute for Quantum Physics, 1-9-1 Kyoyama, Okayama 700-0015, Japan. \\ Kentaroh Yoshida \\ Theory Division, Institute of Particle and Nuclear Studies, \\ High Energy Accelerator Research Organization (KEK), Tsukuba, Ibaraki 305-0801, Japan.
}

(Dated: November 8, 2018)

\begin{abstract}
We discuss an open supermembrane in the presence of a constant three-form. The boundary conditions to ensure the $\kappa$-invariance of the action lead to possible Dirichlet branes. It is shown that a noncommutative (NC) M5-brane is possible as a boundary and the self-duality condition that the flux on the world-volume satisfies is derived from the requirement of the $\kappa$-symmetry. We also find that the open supermembrane can attach to each of infinitely many M2-branes on an M5-brane, namely a strong flux limit of the NC M5-brane.
\end{abstract}

PACS numbers: 11.25.Yb, 11.25.Uv, 11.25.-w, 11.25.Wx

Open supermembranes [1] are attractive objects. Supermembranes [2] are considered as fundamental objects of M-theory [3], which is believed to be the unified theory of superstrings, and open supermembranes are especially interesting because of connection to some realistic phenomenological models via the Horava-Witten scenario [4]. It is well-known that an open supermembrane in flat spacetime can attach on Dirichlet $p$-branes with $p=1,5$ and $9[5,6]$. The $p=5$ case corresponds to M5-brane and the case with $p=9$ is nothing but the end of the world nine-brane discussed in the Horava-Witten theory 4]. As a recent progress, open M5-branes have also been discussed in [7].

It is interesting to consider a generalization of studies of open supermembranes by including constant threeform gauge field (gauge field condensate). In this direction an approach based on the light-cone gauge, with which supermembrane theory is well studied, is not desirable because the light-cone coordinates should inevitably be Neumann directions and furthermore one cannot consider arbitrary electric fluxes. Thus a covariant approach is more appropriate for our purpose [8]. In this letter we basically follow the covariant procedure utilized in [5]. This type of procedure was used to study D-branes of Green-Schwarz (GS) string theories in [9] .

We discuss NC M5-branes from the requirement of the $\kappa$-symmetry of a covariant GS action of an open supermembrane with constant fluxes. In case of open supermembrane, the surface terms appear under the $\kappa$-variation of the action and hence they should be deleted by imposing some appropriate boundary conditions. The usual NC M5-branes are surely included among the conditions, for which the self-duality condition of the flux on the worldvolume is also obtained from the condition that the projection operator should satisfy. In addition, $p=2$ case is

\footnotetext{
*Electronic address: makoto sakaguchi@pref.okayama.jp
}

$\dagger$ Electronic address: kyoshida@post.kek.jp possible from the argument of the boundary conditions based on the $\kappa$-symmetry. As noted later, we should be careful for the interpretation of this configuration. It can be supported just as a strong flux limit of NC M5-branes and this $p=2$ case may describe infinitely many M2branes on the M5-brane.

The covariant GS action of a supermembrane in elevendimensional flat spacetime was given by [11] and it is composed of the Nambu-Goto (NG) part and the WessZumino (WZ) part

$$
S=\int_{\Sigma} d^{3} \xi\left[\mathcal{L}_{\mathrm{NG}}+\mathcal{L}_{\mathrm{WZ}}\right]
$$

where $\Sigma$ denotes the three-dimensional membrane worldvolume with coordinates: $\xi^{i}=\left(\xi^{0}=\tau, \xi^{1}, \xi^{2}\right)$. The NG part is given by

$$
\begin{gathered}
\mathcal{L}_{\mathrm{NG}}=-\sqrt{-g(X, \theta)}, \quad g_{i j}=E_{i}^{A} E_{j}^{B} \eta_{A B}, \\
E_{i}^{A}=\partial_{i} X^{A}-i \bar{\theta} \Gamma^{A} \partial_{i} \theta,
\end{gathered}
$$

and the WZ part is

$$
\begin{aligned}
\mathcal{L}_{\mathrm{WZ}}= & \epsilon^{i j k}\left[-\frac{1}{6} \partial_{i} X^{A} \partial_{j} X^{B} \partial_{k} X^{C} \mathcal{H}_{A B C}\right. \\
& +\frac{i}{2} \bar{\theta} \Gamma_{A B} \partial_{i} \theta \partial_{j} X^{A} \partial_{k} X^{B} \\
& +\frac{1}{2} \bar{\theta} \Gamma_{A B} \partial_{i} \theta \bar{\theta} \Gamma^{A} \partial_{j} \theta \partial_{k} X^{B} \\
& \left.-\frac{i}{6} \bar{\theta} \Gamma_{A B} \partial_{i} \theta \bar{\theta} \Gamma^{A} \partial_{j} \theta \bar{\theta} \Gamma^{B} \partial_{k} \theta\right],
\end{aligned}
$$

where $\mathcal{H} \equiv C-d b$ with the three-form gauge potential $C$ and the two-form gauge potential $b$ on the brane. $X^{A}(A=0,1, \cdots, 10)$ denote eleven-dimensional coordinates.

To balance physical degrees of freedom, the action must be invariant under the $\kappa$-variation,

$$
\delta_{\kappa} X^{A}=-i \bar{\theta} \Gamma^{A} \delta_{\kappa} \theta .
$$


In the open membrane case, we should be careful with the surface terms from the variation, while the non-surface terms vanish for a supergravity solution. They appear only from the WZ part and so it is only for us to consider the $\kappa$-variation of the WZ part.

With a non-trivial constant $\mathcal{H}$ along the $\mathrm{M} p$-brane worldvolume, the bosonic variables $X$ should satisfy the boundary conditions at $\partial \Sigma[12$

$$
n^{i} \partial_{i} X^{\bar{A}}+\epsilon^{i j k} n_{i} \mathcal{H}^{\bar{A}}{ }_{\bar{B} \bar{C}} \partial_{j} X^{\bar{B}} \partial_{k} X^{\bar{C}}=0,
$$

for Neumann directions $\bar{A}_{a}(a=0, \cdots, p)$, and

$$
\partial_{\tau} X^{\underline{A}}=\partial_{t} X^{\underline{A}}=0,
$$

for Dirichlet directions $\underline{A}_{a}(a=p+1, \cdots, 10)$. Here $n^{i}$ is a normal vector to the boundary $\partial \Sigma$, and the subscript $t$ implies a spatial direction on $\partial \Sigma$. With the boundary conditions, the surface terms are given by

$$
\begin{aligned}
\delta_{\kappa} S_{\mathrm{WZ}}= & \int_{\partial \Sigma} d^{2} \xi\left[\mathcal{L}^{(2)}+\mathcal{L}^{(4)}+\mathcal{L}^{(6)}\right], \\
\mathcal{L}^{(2)}= & -i\left[\bar{\theta} \Gamma_{\bar{A} \bar{B}} \delta_{\kappa} \theta+\mathcal{H}_{\bar{A} \bar{B} \bar{C}} \bar{\theta} \Gamma^{\bar{C}} \delta_{\kappa} \theta\right] \dot{X}^{\bar{A}} X^{{ }^{\prime} \bar{B}}, \\
\mathcal{L}^{(4)}= & {\left[-\frac{3}{2} \bar{\theta} \Gamma^{A} \delta_{\kappa} \theta \bar{\theta} \Gamma_{A \bar{B}}+\frac{1}{2} \bar{\theta} \Gamma_{A \bar{B}} \delta_{\kappa} \theta \bar{\theta} \Gamma^{A}\right] } \\
& \times\left(\theta^{\prime} \dot{X}^{\bar{B}}-\dot{\theta} X^{\prime \bar{B}}\right) \\
\mathcal{L}^{(6)}= & \frac{i}{6}\left[\bar{\theta} \Gamma_{A B} \dot{\theta} \bar{\theta} \Gamma^{A} \theta^{\prime} \bar{\theta} \Gamma^{B} \delta_{\kappa} \theta\right. \\
& -\bar{\theta} \Gamma_{A B} \theta^{\prime} \bar{\theta} \Gamma^{A} \dot{\theta} \bar{\theta} \Gamma^{B} \delta_{\kappa} \theta \\
& \left.-2 \bar{\theta} \Gamma_{A B} \delta_{\kappa} \theta \bar{\theta} \Gamma^{A} \dot{\theta} \bar{\theta} \Gamma^{B} \theta^{\prime}\right]
\end{aligned}
$$

where $\dot{X}=\partial_{\tau} X$ and $X^{\prime}=\partial_{t} X . \mathcal{L}^{(n)}$ denotes the terms of $n$-th order in $\theta$.

To ensure the $\kappa$-invariance, (11), (2) and (3) should be deleted by imposing some conditions on $\theta$ called "gluing conditions". Let us recast surface terms to simplify our analysis, before going to the detail.

First, with the Fierz identity

$$
\left(C \Gamma_{A B}\right)_{(\alpha \beta}\left(C \Gamma^{A}\right)_{\gamma \delta)}=0,
$$

we rewrite $\mathcal{L}^{(6)}$ as

$$
\mathcal{L}^{(6)}=\frac{i}{3}\left[\bar{\theta} \Gamma_{A B} \dot{\theta} \bar{\theta} \Gamma^{A} \theta^{\prime}-\bar{\theta} \Gamma_{A B} \theta^{\prime} \bar{\theta} \Gamma^{A} \dot{\theta}\right] \bar{\theta} \Gamma^{B} \delta_{\kappa} \theta .
$$

By using (41) again $\mathcal{L}^{(6)}$ should vanish, and thus does not affect any boundary conditions.

Next supposing that $\mathcal{L}^{(2)}=0$, one can derive

$$
\begin{aligned}
& \bar{\theta} \Gamma^{\bar{A}} \delta_{\kappa} \theta \bar{\theta} \Gamma_{\bar{A} \bar{B}} \theta^{\prime}+\bar{\theta} \Gamma_{\bar{A} \bar{B}} \delta_{\kappa} \theta \bar{\theta} \Gamma^{\bar{A}} \theta^{\prime} \\
= & -\mathcal{H}_{\bar{A} \bar{B} \bar{C}} \bar{\theta} \Gamma^{\bar{A}} \delta_{\kappa} \theta \bar{\theta} \Gamma^{\bar{C}} \theta^{\prime}-\mathcal{H}_{\bar{A} \bar{B} \bar{C}} \bar{\theta} \Gamma^{\bar{C}} \delta_{\kappa} \theta \bar{\theta} \Gamma^{\bar{A}} \theta^{\prime}=0 .
\end{aligned}
$$

Then with (4) and (15) $\mathcal{L}^{(4)}$ is rewritten as

$$
\begin{aligned}
\mathcal{L}^{(4)}=- & \frac{1}{2}\left[\bar{\theta} \Gamma \underline{A} \delta_{\kappa} \theta \bar{\theta} \Gamma_{\underline{A} \bar{B}}+\bar{\theta} \Gamma_{\underline{A} \bar{B}} \delta_{\kappa} \theta \bar{\theta} \Gamma^{\underline{A}}\right] \times \\
& \times\left(\theta^{\prime} \dot{X}^{\bar{B}}-\dot{\theta} X^{\prime \bar{B}}\right) .
\end{aligned}
$$

Thus we have to impose such boundary conditions that delete $\mathcal{L}^{(2)}$ in (11) and $\mathcal{L}^{(4)}$ in (6). The conditions lead to possible configurations of Dirichlet branes. Let us discuss below what configurations are allowed as consistent boundaries.

We shall consider a Dirichlet $p$-brane by imposing a gluing condition for the fermionic variable $\theta$ on the boundary with the gluing matrix $M$

$$
\theta=M \theta, \quad M=\ell \Gamma^{\bar{A}_{0} \bar{A}_{1} \cdots \bar{A}_{p}}, \quad \ell^{2}(-1)^{\left[\frac{p+1}{2}\right]} s=1,
$$

where $s=-1$ when $0 \in\left\{\bar{A}_{0}, \bar{A}_{1}, \cdots, \bar{A}_{p}\right\}$ and $s=1$ otherwise. The matrix $M$ should satisfy $M^{2}=1$. Here we are considering a gluing matrix consisting of a product of gamma matrices for the boundary condition, hence the condition is nothing but $1 / 2$ supersymmetric condition. We may consider, as we will do really, various types of gluing matrices, for example, those composed of a sum of products of gamma matrices.

We shall first examine $\mathcal{L}^{(4)}$, which is independent of the fluxes. For $\mathcal{L}^{(4)}=0$, either of the two relations

$$
\bar{\theta} \Gamma_{\underline{A} \bar{B}} \delta_{\kappa} \theta=0 \quad \text { or } \quad \bar{\theta} \Gamma \underline{C} \delta_{\kappa} \theta=0,
$$

should be satisfied, and we can easily show that these imply

$$
p=2,3 \bmod 4 \quad \text { or } \quad p=1,2 \bmod 4,
$$

respectively. Then it is turn to consider $\mathcal{L}^{(2)}$, which depends on the fluxes. In the case with $\mathcal{H} \equiv 0, \bar{\theta} \Gamma_{\bar{A} \bar{B}} \delta_{\kappa} \theta=$ 0 is necessary for $\mathcal{L}^{(2)}=0$. It can be easily shown that

$$
\bar{\theta} \Gamma_{\bar{A} \bar{B}} \delta_{\kappa} \theta=0 \quad \text { for } p=1,4 \bmod 4 .
$$

Thus we must choose $p=1 \bmod 4$.

In the case with the fluxes, one might naively impose the additional condition $\bar{\theta} \Gamma^{\bar{C}} \delta_{\kappa} \theta=0$. It can be however shown that

$$
\bar{\theta} \Gamma^{\bar{C}} \delta_{\kappa} \theta=0 \quad \text { for } p=3,4 \bmod 4
$$

and this condition cannot be imposed together with $p=$ $1 \bmod 4$.

\section{M2-brane with a critical flux from NC M5-brane}

We will show that the $p=2$ case is possible under a special circumstance, though it seems to be a bit surprising. This case is so special that the second term in (1) can be rewritten as

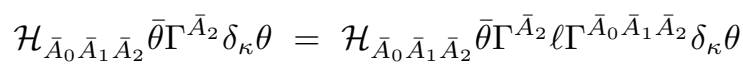

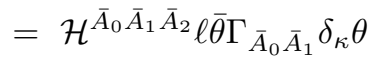

and thus $\mathcal{L}^{(2)}$ vanishes when

$$
1+\ell \mathcal{H}^{\bar{A}_{0} \bar{A}_{1} \bar{A}_{2}}=0 .
$$


$\mathcal{H}$ should be real so that $\ell$ is real and $s=-1$. It follows from (7) that $\mathcal{L}^{(4)}$ disappears. Thus the M2 with the critical flux $\mathcal{H}$ (8) seems to be possible from the $\kappa$ symmetry argument.

How should we interpret this configuration and the special value of the flux? The answer is as follows. It should be considered as a strong magnetic flux limit of a NC M5-brane. In this limit, the self-duality condition of the flux fixes the electric flux at a critical value, and the NC M5-brane may be seen as an M5-brane with infinitely many M2-branes. This is an analogy to D2-brane with the infinitely large magnetic flux, which can be seen as a D2-brane with infinitely many D0-branes. It would be thus plausible to consider it as one of the infinitely many M2-branes. Then the special value of the flux can be naturally understood. In fact, if we consider the M2-brane without a support of an M5-brane, then we confront some problems in explaining the charge conservation law 1] and type IIA string description.

Our analysis considers whether the boundary conditions are Neumann or Dirichlet. In fact, three Neumann boundaries are replaced by the Dirichlet ones in the strong flux limit. According to this, the $p=2$ case is possible in our analysis. However, our analysis does not exclude the possibility that these exists the M5-brane behind the M2-brane.

In order to confirm the above observation we next show the $p=2$ case is indeed realized as a strong flux limit of a NC M5-brane.

\section{Noncommutative M5-brane}

We shall discuss NC M5-branes with fluxes. In comparison to a constant NS-NS two-form in string theory, the three-form on the M5-brane world-volume should satisfy a condition related to the self-dual decomposition of the flux as argued by Seiberg and Witten [10]. As we will see below, this condition appears from the $\kappa$-invariance.

We will consider two types of gluing matrices, (9) and (14), as the cases (A) and (B) respectively. Both of them lead to a commutative M5-brane in the limit the flux goes to zero. In the strong flux limit the condition for $p=2$ is reproduced and it should imply that the open membrane is attaching on one of the infinitely many M2-branes on the M5-brane.

Case (A): The gluing matrix is

$$
M=h_{0} \Gamma^{\bar{A}_{0} \bar{A}_{1} \cdots \bar{A}_{5}}+h_{1} \Gamma^{\bar{A}_{0} \bar{A}_{1} \bar{A}_{2}}
$$

For the condition $M^{2}=1$, which should be satisfied by the gluing matrix, we require that

$$
\begin{aligned}
& -s_{0} h_{0}^{2}-s_{1} h_{1}^{2}=1 \\
& s_{0}= \begin{cases}-1 & 0 \in\left\{\bar{A}_{0}, \bar{A}_{1}, \cdots, \bar{A}_{5}\right\}, \\
+1 & \text { otherwise }\end{cases} \\
& s_{1}= \begin{cases}-1 & 0 \in\left\{\bar{A}_{0}, \bar{A}_{1}, \bar{A}_{2}\right\} \\
+1 & \text { otherwise }\end{cases}
\end{aligned}
$$

First, we examine $\mathcal{L}^{(2)}$. After some algebra, we see that $\mathcal{L}^{(2)}$ vanishes when

$$
h_{1}=\mathcal{H}_{\bar{A}_{0} \bar{A}_{1} \bar{A}_{2}}, \quad h_{1}=h_{0} \mathcal{H}^{\bar{A}_{3} \bar{A}_{4} \bar{A}_{5}} .
$$

For reality of $\mathcal{H}$, we choose $s_{0}=-1$. The condition (10) is nothing but the self-dual condition of the gauge field on the M5-brane [10]

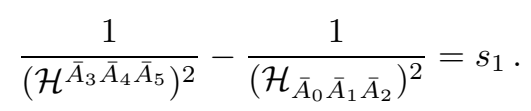

Here it should be remarked that the self-dual condition has been derived from the $\kappa$-invariance. It would be plausible because the $\kappa$-symmetry of open supermembrane should be related to the M5-brane equation of motion 13] and the self-duality 14]. For example, the solution is given by

$$
\mathcal{H}_{\bar{A}_{0} \bar{A}_{1} \bar{A}_{2}}=h, \quad \mathcal{H}^{\bar{A}_{3} \bar{A}_{4} \bar{A}_{5}}=\frac{h}{\sqrt{1+s_{1} h^{2}}} .
$$

It is easy to see that $\bar{\theta} \Gamma \underline{A} \delta_{\kappa} \theta=0$ so that $\mathcal{L}^{(4)}$ vanishes and thus we have found a supersymmetric configuration of a

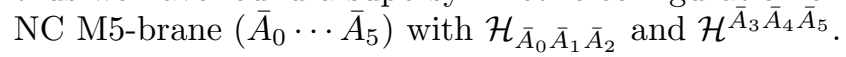

Let us examine the two limits: 1) $\mathcal{H} \rightarrow \infty$ and 2) $\mathcal{H} \rightarrow$ 0 . The flux $\mathcal{H}$ is assumed to be real, so it is sufficient to examine $\left(s_{0}, s_{1}\right)=(-1, \pm 1)$.

As the first example, we consider the case with $s_{1}=$ -1 , say, the NC M5 (012345) with $\mathcal{H}_{012}$ and $\mathcal{H}^{345}$. The conditions (10) and (11) are solved by

$$
\begin{aligned}
& h_{0}=\cos \varphi, \quad h_{1}=\sin \varphi \\
& \mathcal{H}_{012}=\sin \varphi, \quad \mathcal{H}^{345}=\tan \varphi
\end{aligned}
$$

with $0 \leq \varphi \leq \pi / 2$, so that

$$
M=\mathrm{e}^{\varphi \Gamma^{345}} \Gamma^{012345} .
$$

This form is very similar to the special solution found in [5] and it would have some relation with it. For $\varphi \rightarrow 0$, it reduces to the commutative M5 (012345). On the other hand, for $\varphi \rightarrow \pi / 2$, we see that $\mathcal{H}^{345} \rightarrow \infty$ and so it reduces to the previous condition for the $p=2$ case. Thus this result strongly support our previous observation that the $p=2$ case is one of infinitely many M2-branes on the M5-brane. This limit is nothing but the OM limit discussed in 15] with an appropriate scaling of the variables to make the tension finite. 
The second example is the case with $s_{1}=1$, the NC M5 (012345) with $\mathcal{H}_{345}$ and $\mathcal{H}^{012}$. The conditions (10) and (11) are solved by

$$
\begin{aligned}
& h_{0}=\cosh \varphi, \quad h_{1}=\sinh \varphi \\
& \mathcal{H}_{345}=\sinh \varphi, \quad \mathcal{H}^{012}=\tanh \varphi
\end{aligned}
$$

with $0 \leq \varphi<\infty$, so that

$$
M=\cosh \varphi \Gamma^{012345}+\sinh \varphi \Gamma^{345}=\mathrm{e}^{\varphi \Gamma^{012}} \Gamma^{012345} .
$$

For $\varphi \rightarrow 0$, it reduces to the commutative M5 (012345) since $h_{0}=1, h_{1}=0$ and $\mathcal{H}_{345}=\mathcal{H}^{012}=0$. On the other hand, for $\varphi \rightarrow \infty$, the boundary condition $\theta=M \theta$ is rewritten as

$$
2 \mathrm{e}^{-\varphi} \theta=\left[\left(1+\mathrm{e}^{-2 \varphi}\right) \Gamma^{012345}+\left(1-\mathrm{e}^{-2 \varphi}\right) \Gamma^{012} \Gamma^{012345}\right] \theta,
$$

which reduces to $\theta=\Gamma^{012} \theta$ in $\varphi \rightarrow \infty$. Hence the previous condition for the $p=2$ case is reproduced again.

Case (B): We consider the gluing matrix

$$
M=h_{0} \Gamma^{\bar{A}_{0} \bar{A}_{1} \bar{A}_{2}}+h_{1} \Gamma^{\bar{A}_{3} \bar{A}_{4} \bar{A}_{5}},
$$

where $0 \notin\left\{\bar{A}_{3}, \bar{A}_{4}, \bar{A}_{5}\right\}$ is assumed without loss of generality. For $M^{2}=1$,

$$
\begin{aligned}
& -s h_{0}^{2}-h_{1}^{2}=1, \\
& s= \begin{cases}-1 & 0 \in\left\{\bar{A}_{0}, \bar{A}_{1}, \bar{A}_{2}\right\} \\
+1 & \text { otherwise }\end{cases}
\end{aligned}
$$

should be satisfied. Noting the relations,

$$
\bar{\theta} \Gamma_{\bar{A}_{0}} \delta_{\kappa} \theta=h_{0} \bar{\theta} \Gamma^{\bar{A}_{1} \bar{A}_{2}} \delta_{\kappa} \theta, \quad \bar{\theta} \Gamma_{\bar{A}_{0} \bar{A}_{3}} \delta_{\kappa} \theta=0
$$

one can easily find that $\mathcal{L}^{(2)}$ vanishes when

$$
h_{0} \mathcal{H}^{\bar{A}_{0} \bar{A}_{1} \bar{A}_{2}}=-1, \quad h_{1} \mathcal{H}^{\bar{A}_{3} \bar{A}_{4} \bar{A}_{5}}=-1 .
$$

For reality of $\mathcal{H}$ we choose $s=-1$. The condition (15) with (16) also leads to the self-dual condition 10]

$$
\frac{1}{\left(\mathcal{H}^{\bar{A}_{0} \bar{A}_{1} \bar{A}_{2}}\right)^{2}}-\frac{1}{\left(\mathcal{H}^{\bar{A}_{3} \bar{A}_{4} \bar{A}_{5}}\right)^{2}}=1 .
$$

The solution is given by the type of (12) again. It is straightforward to see that $\mathcal{L}^{(4)}$ vanishes for this solution and thus we have found a supersymmetric configuration of a NC M5-brane.

Let us consider the large/small $\mathcal{H}$ limits of the brane. For $\mathcal{H}$ to be real, $\mathcal{H}^{\bar{A}_{0} \bar{A}_{1} \bar{A}_{2}}$ has to be electric. We consider the NC M5 (012345) with $\mathcal{H}^{012}$ and $\mathcal{H}^{345}$. Equations (15) and (16) are solved by

$$
\begin{aligned}
& h_{0}=\cosh \varphi, \quad h_{1}=\sinh \varphi, \quad 0 \leq \varphi<\infty \\
& \mathcal{H}^{012}=-\frac{1}{\cosh \varphi}, \quad \mathcal{H}^{345}=-\frac{1}{\sinh \varphi}
\end{aligned}
$$

so the gluing matrix is

$$
M=\cosh \varphi \Gamma^{012}+\sinh \varphi \Gamma^{345}=\mathrm{e}^{-\varphi \Gamma^{012345}} \Gamma^{012} .
$$

For $\varphi \rightarrow 0, \mathcal{H}^{345} \rightarrow-\infty$ and M2 (012) with $\mathcal{H}^{012}=-1$ is left. On the other hand, for $\varphi \rightarrow \infty$, since all the components of $\mathcal{H}$ become zero and the boundary condition reduces to $\theta=\Gamma^{012345} \theta$, we obtain a commutative M5-brane.

Eventually, the two cases (A) and (B), which are seemingly different, are shown to be equivalent. The condition with (17) can be rewritten as

$$
\begin{aligned}
& \theta=M_{d} \theta, \quad M_{d}^{2}=1 \\
& M_{d}=\operatorname{coth} \varphi \Gamma^{012345}+\frac{1}{\sinh \varphi} \Gamma^{345}
\end{aligned}
$$

so $M_{d}$ is another solution of (10). Thus we find that (13) and (17) describe the same NC M5-brane in different parameterizations. This may support the possibility that the projection operator to describe a NC M5-brane would be unique as denoted in [5], though there is no proof at present. It is nice to try to proof the uniqueness.

So far we have discussed NC M5-branes and M2-branes on the M5-branes as a strong flux limit of the NC M5branes. We can also consider intersecting NC M5-branes from the viewpoint of $\kappa$ symmetry, together with the idea of [9]. We will report on this issue in [16]. It would also be interesting to study the Poisson bracket structure in the strong flux limit along the line of 12, 17, 18. Here we have discussed an open supermembrane in a strong flux limit. If we consider a closed supermembrane instead of an open membrane in this limit, the analysis is boiled down to that of non-relativistic limit [19] of supermembrane [20]. It is interesting to study the relation between non-relativistic membrane and OM theory. It is also interesting to generalize the study in this direction to the AdS backgrounds [21].

\section{Acknowledgments}

We would like to thank Seiji Terashima for variable comments and helpful discussion. This work is supported in part by the Grant-in-Aid for Scientific Research (No. 17540262 and No. 17540091) from the Ministry of Education, Science and Culture, Japan. The work of K. Y. is supported in part by JSPS Research Fellowships for Young Scientists. 
[1] A. Strominger, Phys. Lett. B $\mathbf{3 8 3}$ (1996) 44 arXiv:hep-th/9512059.

[2] B. de Wit, J. Hoppe and H. Nicolai, Nucl. Phys. B 305 (1988) 545.

[3] T. Banks, W. Fischler, S. H. Shenker and L. Susskind, Phys. Rev. D 55 (1997) 5112 arXiv:hep-th/9610043.

[4] P. Horava and E. Witten, Nucl. Phys. B 460 (1996) 506 arXiv:hep-th/9510209; Nucl. Phys. B 475 (1996) 94 arXiv:hep-th/9603142.

[5] K. Ezawa, Y. Matsuo and K. Murakami, Phys. Rev. D 57 (1998) 5118 arXiv:hep-th/9707200.

[6] B. de Wit, K. Peeters and J. C. Plefka, Nucl. Phys. Proc. Suppl. 68 (1998) 206 arXiv:hep-th/9710215.

[7] E. A. Bergshoeff, G. W. Gibbons and P. K. Townsend, "Open M5-branes," arXiv:hep-th/0607193

[8] For open supermembranes on pp-wave and AdS, see M. Sakaguchi and K. Yoshida, Nucl. Phys. B 681 (2004) 137 arXiv:hep-th/0310035; Nucl. Phys. B 714 (2005) 51 arXiv:hep-th/0405109; Nucl. Phys. B 676 (2004) 311 arXiv:hep-th/0306213;

K. Sugiyama and K. Yoshida, Nucl. Phys. B 644 (2002) 113 arXiv:hep-th/0206070.

[9] N. D. Lambert and P. C. West, Phys. Lett. B 459 (1999) 515 arXiv:hep-th/9905031.

[10] N. Seiberg and E. Witten, JHEP 9909 (1999) 032 arXiv:hep-th/9908142.
[11] E. Bergshoeff, E. Sezgin and P. K. Townsend, Phys. Lett. B 189 (1987) 75; Ann. Phys. 185 (1988) 330.

[12] E. Bergshoeff, D. S. Berman, J. P. van der Schaar and P. Sundell, Nucl. Phys. B 590 (2000) 173 arXiv:hep-th/0005026.

[13] C. S. Chu and E. Sezgin, JHEP 9712 (1997) 001 arXiv:hep-th/9710223.

[14] P. S. Howe, E. Sezgin and P. C. West, Phys. Lett. B 400 (1997) 255 arXiv:hep-th/9702111; Phys. Lett. B 399 (1997) 49 arXiv:hep-th/9702008;

[15] R. Gopakumar, S. Minwalla, N. Seiberg and A. Strominger, JHEP 0008 (2000) 008 arXiv:hep-th/0006062.

[16] M. Sakaguchi and K. Yoshida, in preparation.

[17] S. Kawamoto and N. Sasakura, JHEP 0007 (2000) 014 arXiv:hep-th/0005123.

[18] I. Rudychev, JHEP 0104 (2001) 015 arXiv:hep-th/0101039.

[19] J. Gomis and H. Ooguri, J. Math. Phys. 42 (2001) 3127 arXiv:hep-th/0009181.

[20] J. Gomis, K. Kamimura and P. K. Townsend, JHEP 0411 (2004) 051 arXiv:hep-th/0409219.

[21] M. Sakaguchi and K. Yoshida, "Non-Relativistic AdS Branes and Newton-Hooke Superalgebra," arXiv:hep-th/0605124 